\title{
Russie : pauvreté de masse et stigmatisation des pauvres (Partie 1)
}

\section{Karine Clément}

\section{(2) OpenEdition \\ 1 Journals}

Édition électronique

URL : http://journals.openedition.org/conflits/163

DOI : 10.4000/conflits. 163

ISSN : $1777-5345$

Éditeur :

CCLS - Centre d'études sur les conflits lilberté et sécurité, L'Harmattan

Édition imprimée

Date de publication : 1 septembre 1999

ISBN : 2-7384-8616-9

ISSN : 1157-996X

\section{Référence électronique}

Karine Clément, «Russie : pauvreté de masse et stigmatisation des pauvres (Partie 1) 》, Cultures \& Conflits [En ligne], 35 | automne 1999, mis en ligne le 13 mars 2006, consulté le 30 mars 2021. URL http://journals.openedition.org/conflits/163 ; DOI : https://doi.org/10.4000/conflits. 163

Ce document a été généré automatiquement le 30 mars 2021.

Creative Commons License 


\title{
Russie : pauvreté de masse et stigmatisation des pauvres (Partie 1)
}

\author{
Karine Clément
}

Phénomène ancien, la pauvreté en Russie a soudainement suscité l'intérêt des médias nationaux et internationaux depuis la crise financière d'août 1998. Le danger qui touchait désormais les fameuses classes moyennes, symbole des réformes, est pourtant le seul fait nouveau capable d'expliquer cette attention médiatique. Car cette crise n'a en réalité fait qu'aggraver la précarité dans laquelle vit aujourd'hui la majorité de la population, à l'exception d'une mince couche de dirigeants et d'hommes d'affaires influents ${ }^{1}$.

Bien que l'appauvrissement n'épargne aucune catégorie sociale, ce sont les familles nombreuses, les femmes seules avec enfants, les personnes âgées, les enfants et les chômeurs qui comptent parmi les plus exposés au risque de pauvreté. Ce sont aussi des populations dépendantes dans une large mesure des transferts sociaux pour leur survie. Leur chute dans la pauvreté est donc accélérée par la dislocation du système de redistribution et de protection sociale.

Néanmoins, et c'est un élément essentiel dans l'appréhension du phénomène, il n'y a pas de ligne de partage nette et stable entre les catégories sociales exposées et celles qui seraient plus à l'abri de la paupérisation. Ce serait fausser l'image de la réalité sociale que de dresser le portrait des "pauvres", d'en faire une catégorie figée et justiciable de caractéristiques communes. Hormis pour une minorité d'individus, la pauvreté n'est pas un état pour la plupart des Russes qui en font l'expérience, mais un processus de déstabilisation, une vie marquée par l'incertitude du lendemain. Il importe en particulier de rompre avec le stéréotype largement répandu qui oppose les "gagnants» aux "perdants» de la «transition", ceux qui auraient su saisir les occasions offertes par les réformes et ceux qui auraient été incapables ne serait-ce que de s'adapter. Aujourd'hui, en Russie, aucune situation n'est à jamais acquise, sauf pour quelques uns suffisamment pourvus en relations avec les dirigeants politiques ou économiques influents. Les travailleurs, avec ou sans emploi fixe, sont de ce fait loin d'être épargnés. Ils représentent même la majorité des pauvres, au sens que nous 
attribuons à ce terme, à l'issue de notre effort pour cerner la réalité sociale de la pauvreté vécue ${ }^{2}$

Au-delà des images médiatiques et de l'évaluation officielle du phénomène, cet article entend mettre en évidence la réalité de la pauvreté dans toutes ses dimensions, à la fois historiques (appréciation de la nouveauté du phénomène), symboliques (en tant que construction médiatique, politique, scientifique et sociale), subjectives (la pauvreté telle qu'elle est ressentie et vécue) et objectives (l'expérience concrète de la pauvreté).

Ces différentes dimensions, complémentaires et antagonistes à la fois, dessinent un phénomène social brouillé, difficile à évaluer mais dont il importe de préciser la configuration et de mettre à jour les mécanismes explicatifs.

Un phénomène nouveau renvoyé dans l'ordre de l'ancien

Comment évaluer la "nouveauté " du phénomène? La question fait sens dans la mesure où les catégories de présent et passé sont aujourd'hui minées en Russie, rattachées trop exclusivement pour la première à un pôle positif, pour la seconde à un pôle négatif. Démêler la pelote d'amalgames et d'anathèmes constitue une des premières tâches pour cerner la réalité d'un phénomène. Dans quelle partie de cet espace historico-symbolique se situe la pauvreté ?

Sans complètement nier l'existence présente de la pauvreté, de nombreux hommes politiques et chercheurs en sciences sociales y voient un héritage du passé voué à s'atténuer avec l'avancée des réformes et la réussite de la "transition». Une démonstration des tenants de cette thèse emprunte l'un ou l'autre de ces trois temps : rétablir l'existence passée d'une pauvreté dissimulée par le système soviétique, présenter sa pérennité comme conjoncturelle ${ }^{3}$ et nier l'aggravation du phénomène. Mais cette interprétation n'est guère plus mise en avant depuis la crise d'août 1998.

L'occultation partielle de la pauvreté par le pouvoir soviétique est avérée par l'absence de mesure statistique ainsi que par le vocabulaire euphémisant officiellement utilisé. Au mot russe signifiant "pauvreté » était en effet préférée une expression suggérant l'idée de catégories sociales «moins bien loties » [maloobespečennye]. Néanmoins, sans pour autant rendre le problème visible et public, le pouvoir soviétique ne l'ignorait pas. La fixation d'un « minimum de subsistance » témoigne de cette préoccupation. Alastair McAuley estime à 35-40\% la proportion de la population soviétique vivant en dessous du niveau officiel de la pauvreté en 1967-68, pourcentage qui tombe à 15-20\% dans les années $70-80^{4}$. Les estimations, quelles qu'elles soient, doivent de toute façon être considérées avec précaution, étant donné la difficulté à mesurer le niveau de vie réel des Soviétiques qui bénéficiaient de nombreux services sociaux gratuits ou à prix très modérés (médecine, garde d'enfants, logement, vacances, loisirs, etc.). Car une sorte d'Etat social, avec toutes ses imperfections et ses dysfonctionnements bureaucratiques, avait été mis en place en URSS et assurait à la majeure partie de la population une protection sociale minimale qui mettait au moins à l'abri des principaux risques de l'existence. Cet Etat avait, de plus, considérablement resserré l'éventail des inégalités sociales au travers des transferts sociaux et de la politique des salaires.

Peut-on dès lors se prononcer sur l'évolution quantitative de la pauvreté ? Il importe d'effectuer une comparaison à partir des mêmes critères. Si nous considérons le montant officiel du budget minimal de subsistance, celui-ci s'élevait en 1989 à 140 roubles par mois, ce qui équivaut à 1.4 millions de roubles en 1996 compte tenu de l'inflation. Un tel chiffre placerait $90 \%$ de la population sous le seuil de pauvreté5. Ainsi, le début des réformes marque incontestablement l'entrée dans une période nouvelle et 
durable d'appauvrissement de la majorité de la population. Il est néanmoins indéniable que la pauvreté préexistait aux réformes. Les mendiants, vagabonds, enfants des rues démentaient l'idéal de la société communiste. Mais, il ne s'agit pas de tirer des conclusions exagérées quant à l'importance de la dissimulation partielle de la pauvreté par les dirigeants soviétiques. La pauvreté telle qu'elle se présente aujourd'hui constitue un fait social nouveau tant par son ampleur que par la diversité des populations touchées. La " transition vers la démocratie de marché » non seulement n'a pas amélioré le sort des "anciens pauvres", mais, de plus, a fragilisé des catégories sociales habituellement considérées comme protégées des risques de pauvreté, voire comme les mieux placées pour profiter de la libéralisation économique (les catégories à haut niveau d'instruction, les travailleurs les plus qualifiés).

La pauvreté en représentation : non-dits, presque dits et distorsions

La nouveauté de la pauvreté réside aussi dans la façon dont elle est aujourd'hui représentée dans l'espace public. Alors que l'idéal démocratique a pris la place du communisme dans les discours officiels et les représentations collectives, on déclare faire toute la lumière sur les chiffres, désormais régulièrement calculés et publiés. Mais, à y regarder de plus près, la question de la pauvreté et des pauvres est-elle posée en des termes vraiment plus explicites et respectueux de la réalité ?

Il apparait en fait que la pauvreté, en tant que catégorie de représentation, continue à faire l'objet d'importants investissements politiques, médiatiques et scientifiques. Elle constitue un enjeu dans les luttes de classement qui opposent différents groupes sociopolitiques dans les représentations qu'ils entendent donner d'eux-mêmes et de la société russe. De la perception que se fait la population de l'étendue et de la configuration du problème de la pauvreté en Russie dépend en effet son attitude à l'égard d'une «transition " présentée comme incontournable. De l'estimation qu'on en fait découle, en partie, la justification ou le discrédit des réformes.

Essayons de décrire les différentes positions qui alimentent le débat. Un premier type de divergence porte sur l'évaluation de l'étendue du phénomène et en particulier sur les méthodes et les critères utilisés. Au sortir de la période soviétique, un certain consensus s'est instauré entre les sociologues, les hommes politiques et les responsables d'organisations internationales pour considérer que les instruments de mesure statistiques hérités du passé soviétique n'étaient plus adaptés ${ }^{6}$. Ce jugement, en partie fondé sur des critiques scientifiques, contenait aussi des prises de positions politiques: tourner le dos au passé, arrimer le pays aux réformes de marché en atténuant, si ce n'est la réalité de leur coût social, du moins sa perception. L'un d'entre eux consiste à contester les modes de calcul statistique, en particulier le niveau du seuil de pauvreté, jugé trop élevé par rapport aux standards occidentaux ${ }^{7}$

. Cette réforme, qui entraînait pourtant une révision radicale de la perception de la pauvreté, n'a guère soulevé de protestations chez les sociologues russes, qui estimaient pour la plupart que « l'aggravation de la pauvreté ne durerait pas plus d'un an et que ce minimum vital (...) ne serait utilisé que pendant cette période ${ }^{8}$. La majorité des chercheurs en sciences sociales se plièrent d'ailleurs à cette révision politique des critères et des méthodes de mesure de la pauvreté. Comment expliquer cet accord tacite pour garder temporairement le silence sur le pauvreté ? Trois raisons peuvent être avancées : un consensus sur la nécessité des réformes, une forte pression politique et une certaine méfiance envers le "peuple». Tout d'abord, les réformes dites « libérales » bénéficiaient à cette époque (de 1992 à 1993) d'un large soutien populaire, 
en partie orchestré par les intellectuels et les hommes politiques. La pauvreté était alors soit considérée comme appelée à disparaitre avec le succès des réformes, soit légitimée, ou banalisée, au nom des indispensables réformes 9 . La voix de ceux qui se plaignent de s'appauvrir, de faire les frais des réformes, devait donc être ignorée, la pauvreté étant présentée comme un mal nécessaire, si ce n'est «normal» dans la conjoncture d'une nécessaire "transition" vers le libre marché. Ce raisonnement repose, en effet, sur le principe de l'irresponsabilité d'un "peuple» incapable d'accepter les réformes d'un bien commun fixé par les dirigeants éclairés du pays et qu'il faut, par conséquent, ignorer au nom de l'efficacité politique. Ce discours sacrificiel, déconstruit et critiqué par nombre de philosophes ${ }^{10}$, étend ses ramifications dans le passé soviétique où il signifiait à la population d'attendre patiemment « l'avenir radieux du communisme ». Mais il ne se réduit pas pour autant à un reliquat du passé, puisqu'il acquiert une nouvelle légitimité à la faveur d'une «transition » donnant au discours une aura démocratique où le gouvernement se présente et apparaît comme le dépositaire du bien du peuple, élu par le peuple et délégué par lui pour veiller à ses intérêts et combattre ses passions ${ }^{11}$.

Ainsi, acceptée et légitimée par la communauté scientifique, la méthode officielle de mesure a eu pour effet, au moins jusqu'à la crise d'août 1998, de diminuer la proportion de personnes vivant en dessous du seuil de pauvretée ${ }^{12}$. En quoi cette évolution statistique reflète-t-elle la réalité ? Il est frappant de constater que cette réduction du nombre officiel de pauvres s'opère dans un contexte de baisse constante des salaires et des revenus réels ${ }^{13}$. De plus, signe supplémentaire de l'aggravation de la pauvreté, la part des dépenses alimentaires dans les dépenses des ménages ne cesse de croître pendant cette période ${ }^{14}$. Ces différents signes font planer un doute sur la fiabilité des pourcentages officiels. Enfin, à la suite de l'inflation qui a suivi la crise financière d'août 1998, le salaire moyen réel a encore une fois chuté de moitié, ce qui n'a relevé le pourcentage de personnes vivant en dessous du seuil de pauvreté qu'à $27.1 \%$. La faible incidence de la crise sur l'étendue de la pauvreté paraît problématique, même si l'on peut supposer que les catégories sociales les plus appauvries avant la crise sont celles qui ont été les moins touchées par le choc financier ${ }^{15}$. Plusieurs facteurs, liés à l'évolution socio-politique du pays, expliquent ce réinvestissement du thème de la pauvreté par les chercheurs. D'une part, le problème est mis en avant par le Parti communiste de Ziouganov qui revient sur le devant de la scène à l'occasion des élections législatives de décembre 1995. De même, les syndicats s'emparent du problème. Ils présentent des estimations dépassant largement les chiffres officiels et dénoncent le scandale d'un Etat incapable d'assurer la survie de la majorité de la population ${ }^{16}$. D'autre part, la pauvreté fait désormais peur et devient une menace subjectivement intégrée par la plupart des individus. Voir les sondages publiés trimestriellement dans Monitoring obšcestvennogo mnenja (le bulletin du VCIOM Centre Russe d'Etude de l'Opinion Publique).]]. Enfin, la pauvreté s'élargit à des couches sociales que les sociologues considéraient comme appelées à profiter des réformes, à savoir l'intelligentsia et les travailleurs qualifiés ${ }^{17}$.

Toutes les remarques précédentes nous conduisent à privilégier les approches les plus adaptées pour saisir le phénomène en creux, sans figer des processus caractérisés par leur caractère mouvant et diffus. Parmi celles-ci, les enquêtes centrées sur le travail, sur les conditions de travail et de rémunération, semblent refléter la réalité telle qu'elle est vécue par la plus grande partie de la population. En effet, les diverses allocations sociales ne garantissant pas le minimum vital, quelle catégorie de la population russe 
peut aujourd'hui survivre sans avoir de travail ? Or, le travail ne met aucunement à l'abri de la pauvreté, au contraire, il soumet le travailleur au risque permanent de sombrer, au moins temporairement, dans la pauvreté ${ }^{18}$. Cette instabilité liée au travail, à la fois matérielle, sociale, professionnelle et psychologique, dessine le trait fondamental du phénomène de la pauvreté telle qu'elle est réellement vécue par la majorité de la population russe ${ }^{19} 20$.

Contre l'approche par le caractère précaire et déstabilisant du travail et de ses revenus, on évoque souvent la sous-estimation des revenus individuels en raison de l'importance des revenus non déclarés issus du troc ou de l'économie informelle (culture du potager, multi-activités, production domestique, ventes dans les rues et autres pratiques de "débrouille»). Mais quelle est au juste la réalité de cette "débrouille» et de ses revenus informels? Le secteur informel représente de 20 à $40 \%$ du PIB selon les différentes estimations.Cette richesse non enregistrée par les statistiques permet, grâce à une seconde ou à une troisième activité, à près de la moitié des travailleurs de survivre malgré la faiblesse de leur revenu officiel ${ }^{21}$. Le décalage entre les revenus déclarés à l'enquête et les dépenses des ménages peut aussi s'expliquer par « l'irrégularité du paiement des salaires, que ce soit dans les entreprises étatiques ou non " ${ }^{22}$. De plus, les revenus intentionnellement dissimulés, conformément aux exigences des employeurs profitant d'une main-d'œuvre non déclarée, concernent surtout «les structures nouvelles, surtout commerciales et financières, concentrées dans quelques grandes villes, et dans lesquelles sont intégrées des couches à haut revenu officiel, alors que la masse de la population reste à l'écart » ${ }^{23}$

<!--SPIP--> . La prise en compte du secteur formel et des pratiques de "débrouille", loin de mettre en cause notre conception de la pauvreté en Russie, vient donc la conforter. En effet, la « débrouille » est surtout faite d'un travail précaire et d'un salaire irrégulier. Telle est en tout cas la réalité vécue par les travailleurs auprès desquels nous avons effectué notre enquête. Dans la majeure partie des cas, la « débrouille » permet de ne pas sombrer dans le dénuement total et la misère. Mais, avant d'être source d'enrichissement ou de réalisation de soi, c'est un combat quotidien pour la survie.

L'activité complémentaire, ou secondaire, apporte-t-elle davantage de possibilités de réalisation de soi? Pour les ouvriers ou techniciens, cette activité suppose une deuxième journée de travail, après celle, plus ou moins longue selon la conjoncture, qu'ils passent dans l'usine. Après y avoir passé quelques heures, quand il n'y a plus rien à faire, beaucoup d'ouvriers demandent souvent rapidement au contremaître la permission de partir. Certains vendent des journaux dans les rues et les couloirs du métro, d'autres tiennent un kiosque sur un marché, d'autres encore réparent des voitures dans un garage, cousent ou bricolent. Mais ils hésitent à quitter complètement l'usine, ce qui les priverait d'un statut certes précaire, mais au moins existant et donnant un droit formel à une certaine protection juridique et sociale. Et c'est aussi, paradoxalement, l'espoir d'une amélioration de leur situation dans l'usine en cas d'une conjoncture plus favorable qui les retient dans cette activité. Beaucoup, enfin, préfèrent conserver un lien avec le travail d'usine, généralement plus qualifié.

La dignité se retrouve-t-elle davantage lorsque l'activité «informelle» est exercée à plein temps? Ce type de « débrouille » peut fournir à certains individus des possibilités d'émancipation, voire d'enrichissement. Il faut aussi relativiser l'emprise de la mafia sur ce petit commerce. Bien que ces réseaux de « débrouille » soient contrôlés par des chefs, ces derniers ne se distinguent absolument pas des dirigeants d'entreprise et 
n'appartiennent d'ailleurs pas tous à la " mafia » au sens strict du terme. Cette sphère de la « débrouille » connaît en effet une organisation du travail non moins hiérarchique que dans l'usine, les rapports hiérarchiques y sont seulement moins formalisés et donc plus propices à l'affirmation de l'arbitraire et du pouvoir des dirigeants du réseau de "débrouille». Dans certains cas, ceux qui s'engagent dans ce type de "débrouille» parviennent à en tirer profit pour accroître la maîtrise de leur vie et de leur travail C'est le cas par exemple de certains celnoki par exemple - ces marchands faisant des navettes entre deux régions ou deux pays afin d'acheter et revendre des marchandises en jouant sur les différences de prix.]].

Que conclure de la « débrouille » au regard de la diversité de ses formes et de ceux qui y recourent? Chaque individu vit en réalité cette expérience de façon singulière, selon son histoire, les attentes qu'il place dans le travail, les objectifs qu'il poursuit. Mais de façon plus générale, il apparaît qu'on ne peut ni valoriser à l'excès la « débrouille », ni la condamner absolument au regard de ce qu'elle apporte à ceux qui choisissent de tenter l'expérience. Elle permet déjà de survivre, voire de vivre mieux. Surtout, elle procure à ceux qui s'y livrent le sentiment, recueilli dans nos entretiens, de " prendre leur destin entre leurs mains ». La notion de " débrouille » vaut donc pour ce qu'elle signifie du point de vue des ouvriers, qui y voient une manière de s'en sortir par leurs propres moyens, une manière de faire preuve de leurs capacités d'adaptation. Néanmoins, cette notion ne vaut, guère d'un point de vue analytique, en ce qu'elle renvoie à des comportements trop largement répandus chez tous ceux qui se trouvent soumis à la nécessité de survivre et de s'adapter à la déstabilisation de leur situation. Et elle n'ajoute rien non plus au concept traditionnel de travail, car la « débrouille » est faite avant tout de travail. Le travail qu'elle désigne est un peu moins formel, mais le travail d'usine lui-même devient de plus en plus informel. Retenons surtout, contre les visions idylliques de la « débrouille » qu'elle n'implique, par rapport au travail d'usine, ni moins d'efforts, ni moins de contraintes, ni moins de précarité et de risque de se retrouver du jour au lendemain démuni de tout. Permet-elle la sortie de la pauvreté ? Il semble que l'appréciation doive se faire mesurée au vu de la relative faiblesse et de la précarité des revenus perçus par les plus démunis désavantagés dans les rapports de force, soumis aux aléas de ce marché et de l'arbitraire des têtes de réseaux qui contrôlent la distribution des tâches et des rémunérations.

Notre approche entend justement analyser l'expérience subjective de la réalité de la pauvreté par les personnes concernées, expérience dominée par l'omniprésence d'un travail précaire, déstabilisant et peu, ou irrégulièrement, rémunérateur.

La perception des pauvres : extrémistes, incapables-coupables ou victimes-exclus

La plupart des intellectuels tendaient à considérer les inégalités sociales entraînées par les réformes libérales de la période 1992-1994 comme justes, car traduisant les mérites des uns et des autres. Ceux qui souffraient des changements étaient perçus comme ayant vécu jusque-là aux crochets du système, grâce à des privilèges sans rapport avec leurs mérites (telle est en particulier l'image donnée de la nomenklatura mais aussi des ouvriers, du "peuple» symbolisé par l'«homo-sovieticus»). A l'inverse, ceux qui bénéficiaient des transformations devaient voir leurs compétences enfin reconnues (comme, par exemple, l'intelligentsia dite "créatrice», les ouvriers qualifiés, les techniciens et les ingénieurs). L'appauvrissement des premiers et l'enrichissement des seconds étaient donc justifiés. Une justification d'autant plus facilitée que nombre d'intellectuels manifestaient une forte méfiance à l'égard d'un "peuple " soupçonné 
d'inconséquence politique. Au début du pouvoir eltsinien (1991-1994), ces derniers étaient ainsi surtout soucieux de traquer et de dénoncer la persistance au sein du peuple de la mentalité " homo sovieticus " ${ }^{24}$. Mais cette méfiance s'estompa quelque peu avec le constat de la passivité politique du "peuple » et sa tolérance aux réformes. Un changement important intervint notamment après que la crise financière d'août 1998 ait frappé la mythique "classe moyenne ", que les réformateurs appelaient de leurs vœux, et à laquelle maints sociologues et journalistes attribuaient toutes les qualités (sens de l'entreprise, goût de l'initiative, dynamisme, intelligence). Les commentateurs firent alors preuve de plus d'humanité dans leur appréciation de la pauvreté, un phénomène qui menaçait désormais les petits chefs d'entreprise, les spécialistes qualifiés, les professions libérales et intellectuelles. Les malheurs du «peuple» ont donc été reconnus, au moins le temps qu'a duré l'onde de choc. L'émotion passée, le ton est redevenu plus méprisant et suspicieux, dans les propos de certains intellectuels ${ }^{25}$ et, surtout, les propos des journalistes ${ }^{26}$. L'on retrouve également, chez certains dirigeants politiques, ce discours méprisant envers les pauvres qui ne se résignent pas ${ }^{27}$.

Le débat public semble centré autour des oppositions modération/extrémisme, tempérance/revanchisme. Il est fait grief aux plus pauvres de ne pas avoir supporté avec suffisamment de patience leur appauvrissement, leur réticence au sacrifice ayant fait échouer les réformes ${ }^{28}$. Quant aux pauvres qui ne manifestent pas collectivement leur mécontentement, comment sont-ils perçus ? Une première catégorie de perception les représente comme des individus incapables d'assumer l'individualité que leur confère la " transition vers le marché ». Ils sont alors stigmatisés pour leur incapacité à surmonter l'appauvrissement. Le discours le plus commun consiste à rendre les victimes elles-mêmes responsables de leur pauvreté, à mettre en doute leur volonté de s'en sortir, leur capacité de saisir les occasions offertes par le marché, leur inadaptation aux réformes. Une expression russe, mi-ironique, mi-proverbiale, résume parfaitement cette opération symbolique: «le sauvetage du naufragé est l'affaire du naufragé luimême ». Cette injonction correspond au nouveau système de normes qui se renforce aujourd'hui en Russie et place l'individu au sommet de la pyramide des valeurs. Rien n'est plus socialement valorisé qu'un individu maitrisant seul sa vie, se façonnant et s'enrichissant par ses seules forces. La reconnaissance de cette norme est vérifiée par les entretiens et la façon dont les travailleurs se définissent subjectivement. Lorsque leur expérience de vie le leur permet, c'est-à-dire lorsque leur situation matérielle et professionnelle demeure relativement stable et assurée, ils mettent surtout en avant leur individualité et rejettent l'idée d'une quelconque appartenance sociale. "Quoi qu'il se produise dans le monde, en économie ou en politique, je suis un homme avec un grand $\mathrm{H}$, une personne à part entière ", «je suis un homme, un individu », «je suis maitre de mon destin », « je suis un individu par moi-même et pour moi-même » ${ }^{29}$.

Ceux qui, à l'inverse, ne bénéficient pas de telles conditions et ne se sentent pas en mesure de satisfaire aux normes individualistes dominantes aujourd'hui - et ils forment la grande majorité - se déprécient. La présentation de soi devient alors un exercice d'auto-humiliation: "Je suis un travailleur qui ne s'appartient pas et qui est dépendant ", "nous sommes des cochons, des gens écrasés, sans rien, sans certitude ", "j'ai été réduit à l'état d'esclave », " nous sommes traités comme du bétail», "je ne peux pas dire que je suis un homme », « je ne suis rien », etc. Si, ainsi, une minorité des travailleurs satisfont à la norme sociétale portée par l'idéologie libérale et se reconnaissent dans l'image d'un individu fier, autonome et sûr de lui, qu'en est-il de 
tous les autres qui vivent leur individualité comme on porte un fardeau? «Je sais bien que je devrais essayer de trouver un autre travail que celui-ci, où je ne suis pas payée. Mais à mon âge, qui me prendra? ». "C'est vrai qu'à la longue, on finira par ne plus supporter, ça ne durera pas éternellement. On ne peut pas s'accrocher comme ça à sa place, soulagé et honteux à la fois que l'autre ait été licencié et pas toi. Un jour, ça va éclater!». Ces travailleurs peuvent être rattachés à ce que R. Castel nomme l'individualisme "négatif » ${ }^{30} \mathrm{ou}$ à ce que nous appellerions l'individualisme de la souffrance. Et, comme Castel, nous souhaitons éviter tout malentendu puisque ceux qui portent leur individualisme dans la souffrance ne sont pas forcément des nostalgiques du paternalisme et ne sont pas obligatoirement tentés par la subordination à des collectifs tutélaires. C'est au contraire leur individualisme qui passe par la dépendance et la soumission. Car ils sont en même temps sommés de construire leur individualité et défavorisés dans les conditions d'accès à l'autonomie individuelle, leur subjectivité étant écrasée par ces deux mouvements contraires et convergents.

Si la plupart des images attachées aux pauvres mettent en cause leur individualité, leur capacité ou leur irresponsabilité, certaines autres les présentent comme des victimes. Les pauvres seraient victimes d'un nouveau système qui ne leur fait pas de place et les rejettent. Certains sociologues s'efforcent en effet de tracer des sous-catégories au sein de la catégorie, jugée "vaste " selon eux, de "pauvres». C'est précisément la justification qu'ils donnent à la constitution de la catégorie d'« exclus», subdivision de celle de pauvres ${ }^{31}$. Dans la perspective d'une analyse de la genèse du concept d'exclusion, aujourd'hui couramment admis en Occident mais qui a mis des années pour s'imposer dans l'espace médiatico-politique et scientifique, il est particulièrement intéressant d'observer le passage de l'une catégorie à l'autre tel qu'il s'est effectué en Russie, en un temps extrêmement court et sur l'initiative évidente des sociologues. L'exclusion n'a donc pas encore éclipsé complètement la notion de pauvreté et les mécanismes qui ont conduit à son apparition s'étalent encore en pleine lumière. La notion a été introduite par les chercheurs en sciences sociales sous l'influence non seulement de leurs collègues occidentaux mais aussi des organisations internationales telles que l'ONU ou la Banque Mondiale ${ }^{32}$. Elle est généralement investie d'un sens opératoire dans le sens où ses adeptes appellent au ciblage de la politique sociale en faveur des exclus, réforme que la Douma se refuse à voter pour le moment, malgré l'insistance du gouvernement ${ }^{33}$.

Aussi, les pauvres, même qualifiés d'exclus, tendent-ils à être tenus eux-mêmes pour responsables de leur situation, déchiffrée comme un échec ou comme résultant d'un manque de bonne volonté, presque d'un choix. Il peut s'agir d'une fainéantise, d'un goût trop marqué pour l'assistanat, d'une réticence à assumer ses responsabilités, sa liberté. Les tenants de cette position, nombreux surtout dans les premières années des réformes, suivent une logique apparemment infaillible : les travailleurs soviétiques ne s'investissaient pas assez dans leur tâche, le système ne les y incitant pas ; les réformes, par l'introduction du chômage et la différenciation des salaires, doivent conduire à responsabiliser les travailleurs et à contrarier leur tendance au moindre effort ${ }^{34}$. Ceux qui n'ont pas changé de comportement à l'égard du travail n'ont que ce qu'ils méritent, ils s'appauvrissent. Les pauvres doivent leur état au fait que non seulement ils ne travaillent pas assez mais en plus se montrent inconséquents et affichent des revendications démesurées. A partir de 1994, ce type de raisonnement est surtout tenu par les journalistes de la presse libérale et par les dirigeants politiques. Les chômeurs surtout sont mis en cause, accusés de paresse ou de parasitisme [idivencestvo]. 
Une autre explication, voire justification, de l'exclusion résiderait dans l'inutilité qui caractérise de plus en plus de travailleurs avec le passage à l'économie de marché. Ceux-ci seraient " en trop ", " superflus » [lišnie]. Une offensive massive est menée par les dirigeants politiques et économiques pour imposer l'idée qu'il existerait des travailleurs inutiles et pour suggérer la nécessité de se débarrasser de leur poids inutile pour l'entreprise, soit par des licenciements, soit par une sous-rémunération ${ }^{35}$. Les sociologues reprennent le qualificatif et multiplient les enquêtes pour tenter de tracer le portrait de ces " travailleurs en trop $"{ }^{36}$. De nombreuses études ont été commandées par le Ministère du Travail afin de mesurer l'étendue du phénomène ${ }^{37}$. Pourtant, certaines analyses démontrent parfaitement que le "surplus de main-d'œuvre" correspond au moins en partie à une stratégie des entrepreneurs qui ont tout intérêt à conserver un volant de main-d'œuvre peu payé et utilisable selon les fluctuations de l'activité ${ }^{38}$.

Dans quelle mesure ces images correspondent à la réalité ? Elles sont émises par des groupes sociaux (dirigeants d'entreprise, gouvernants, intellectuels) qui, pour n'être pas considérés comme complètement légitimes par les travailleurs, n'en disposent pas moins d'un certain crédit au yeux de la population en ce qui concerne sa capacité à déchiffrer le monde moderne et à s'y adapter. Aussi ces images exercent un indéniable effet d'imposition de sens sur la façon dont les travailleurs se représentent eux-mêmes. Elles érigent un univers symbolique bien éloigné de celui que fait apparaître à nos yeux d'observateur les pratiques et activités des travailleurs, marquées au contraire par un surtravail, une utilisation accrue de leur force de travail accompagnée d'une sousrémunération, une modération de leur consommation et une forte retenue à revendiquer de quelconques droits ou garanties. Toutes ces représentations publiques, diverses et déformantes, contribuent en fait à enlever aux "pauvres » la maîtrise de leur image publique. Elles agissent par des modes de dévalorisation, de négation existentielle et de brouillage. Cette dernière modalité domine puisque la catégorie de " pauvres» est investie d'une pluralité d'images souvent contradictoires les unes avec les autres, cette configuration symbolique autorisant à supposer l'existence d'une certaine lutte de classement du phénomène de la pauvreté dans la réalité sociale russe. Cette lutte laisse apparaitre des dominants et des dominés mais elle produit des images trop multiples, trop peu figées, pour que les " pauvres " puissent être considérés, et se considèrent eux-mêmes, comme définitivement classés d'un côté ou de l'autre de l'espace social. Cette relative fluidité et multiplicité ouvre un étroit passage pour une émancipation des " pauvres » à l'égard des images qui leur sont accolées. Cette dernière assertion doit cependant être nuancée par la prise en compte des rapports sociaux concrets et les processus structurels alimentant la construction sociale du phénomène de la pauvreté. Or, les projecteurs sont braqués sur le phénomène en lui-même et laissent dans l'ombre les mécanismes de la paupérisation. Cette sorte d'occultation renforce d'autant plus le poids des images symboliques attachées à la pauvreté et aux pauvres.

$<!-$ SPIP-->

$<$ !--SPIP-->

Que retenir de cette variété hétéroclite et contradictoire des images et représentations attachées à la pauvreté? L'agitation politique, médiatique et intellectuelle, qui accompagne ce thème prouve déjà son caractère stratégique dans l'évolution et l'évaluation de la réalité sociale de ce pays. D'une part, les catégories sous lesquelles le 
thème apparaît dans l'espace public contribuent en grande partie à brouiller la perception que les individus, concernés ou non, peuvent se faire de la pauvreté. Mais, d'autre part, elles sont en même temps constitutives du phénomène. Aussi, s'avère-t-il indispensable de se pencher sur les expériences subjectives de la pauvreté telle qu'elle est vécue et ressentie par les pauvres eux-mêmes.

\section{NOTES}

1. Selon les données officielles du Comité d'Etat aux Statistiques [Goskomstat], 37.7\% de la population vit en dessous du seuil de pauvreté fixé à 924 roubles, selon les recommandations méthodologiques du Ministère du Travail du 10 novembre 1992 (début 1999, un franc français valait quatre roubles). Ce taux paraît à première vue peu élevé dans un pays où le PIB a chuté de 50\% depuis 1991. Mais qu'est-ce qu'un tel chiffre recouvre en réalité ? Le seuil de pauvreté est calculé selon des modalités et des critères fixés par le Ministère du Travail qui fixe un minimum de subsistance sur la base du prix d'un ensemble de produits et services jugés indispensables pour la survie. Il peut donc évoluer en fonction non seulement de la conjoncture mais aussi de l'évolution des critères administratifs de mesure. Depuis 1992, le nombre de denrées constitutives du minimum alimentaire a ainsi été drastiquement réduit. Des réductions similaires ont touché des produits comme les œufs et le lait. A l'origine, il était ainsi prévu qu'un adulte avait besoin de $64 \mathrm{~kg}$ de viande par an, ce chiffre est descendu jusqu'à $27 \mathrm{~kg}$. Cf. Guy Standing, "Societal impoverishment : the challenge for Russian social policy ", Journal of European Social Policy, 8 (1), 1998, pp. 23-42. Depuis lors, la part des produits alimentaires dans les dépenses minimales de subsistance s'est accrue et s'élevait, fin 1998, à 68,3\% des dépenses minimales de subsistance, entraînant une sous-évaluation du renchérissement du minimum vital puisque les prix des produits alimentaires ont augmenté moins rapidement que ceux d'autres postes de dépense difficilement compressibles tels que les vêtements, l'équipement ménager, les services, les soins ou les transports.

2. Cet article repose sur une enquête réalisée de 1994 à 1997 essentiellement auprès de travailleurs, à partir d'entretiens (collectifs et individuels) et d'observations directes. Elle porte sur les travailleurs subalternes d'entreprises (de différentes régions et branches) ainsi que sur des personnes, bien souvent les mêmes, exerçant une activité (secondaire ou principale) dans la sphère des services ou dans l'informel. Parmi eux, un certain nombre était formellement privé d'emploi et beaucoup étaient à la retraite mais toujours en activité. Nous les avons rencontrés dans différentes situations : dans des grandes entreprises privatisées en crise (l'usine automobile ZIL à Moscou, une usine métallurgique à Ekaterinbourg, une usine textile à Tver), dans une grande entreprise privatisée monopolistique et rentable (une usine d'entretien et de construction de gazoducs de la banlieue de Moscou), dans une entreprise étatique en crise appartenant au complexe militaro-industriel (un groupement scientifico-industriel fabriquant des composants électroniques dans la banlieue de Moscou), dans de petites firmes privées 
(dans les secteurs alimentaire et électroménager, à Moscou), et dans divers lieux de " débrouille» (marchés, chantiers, domiciles, datchas, etc.).

3. «La plupart des experts prévoient que les pires dislocations se feront sur le court terme, les transformations conduisant à une amélioration substantielle sur le long terme ", Thomas A. Mroz et Barry M. Popkin, « Poverty and the Economic Transition in the Russian Federation », Economic Development and Cultural Change, 44 (1), oct. 1995, pp. 1-31, p. 1 ; « Notre tâche est de présenter le contexte historico-économique du phénomène et de montrer la pauvreté comme un état économique et sociopsychologique passager », S. I. Lunev et A. I. Salickij, « Bednost' - ne porok ? » [La pauvreté n'est pas vice ?], Vestnik rossijskoj akademii nauk, 66 (6), 1996, p. 83.

4. Alastair McAuley, Economic Welfare in the Soviet Union, Madison, University of Wisconsin Press, 1979, p. 70.

5. Guy Standing, op.cit., p. 25 ; voir aussi Marina Mozhina, « The Poor: What is the Boundary Line », Problems of Economic Transition, 35 (6), oct. 1992, en particulier p. 69 : «Si l'on prend la norme précédemment admise du budget minimum à la consommation et qu'on la calcule dans les prix d'aujourd'hui (...), alors la population entière serait en dessous du seuil de pauvreté ».

6. Ils étaient considérés comme inaptes à mesurer l'étendue de la pauvreté dans la mesure où le seuil de pauvreté fixé correspondait moins à un critère de mesure statistique qu'à un objectif politique et social à poursuivre : l'avancée vers un niveau de consommation minimalement acceptable dans les conditions du système socialiste. Voir, par exemple, J. Klugman et J. Braithwaite, « Poverty in Russia during the Transition : an Overview », The World Bank Observer, 13 (1), 1998, pp. 37-58; A. Atkinson et J. Micklewright, Economic Transformation in Eastern Europe and the Distribution of Income, Cambridge, Cambridge University Press, 1992.

7. «La nouveau seuil de pauvreté a été fixé de 5 à $10 \%$ plus haut qu'il ne l'aurait été si les normes occidentales en matière alimentaire avaient été adoptées ", T.A. Mroz et B.M. Popkin, op.cit., p. 3. Ainsi, la Banque Mondiale a expressément qualifié le chiffre de $90 \%$ de pauvres d'« estimation subjective ». Cf. Banque Mondiale, « Poverty in Russia », World Bank Report, n' 14110-RU, 1995. Et c'est sous son influence et avec son soutien financier que le Ministère du Travail, en août 1992, a réformé les critères de calcul du seuil de pauvreté ainsi que les méthodes d'enquête. De la sorte, le pourcentage de pauvres est passé de $90 \%$ (selon les critères soviétiques) à $33.5 \%$ de la population (selon les critères du Ministère russe).

8. N. Rimachevskaïa, « Paupérisation et exclusion en Russie », Le Courrier des Pays de l'Est, juillet 1998, p. 59.

9. Une telle perception est largement répandue chez les réformateurs libéraux comme

E. Gaïdar ou A. Tchoubaïs qui entendaient arrimer le pays sur la voie de la « démocratie de marché », quel qu'en soit le coût. E. Gaïdar déclarait ainsi en 1993 : « la politique la meilleure est une politique responsable, pas le populisme ». Cf. E. Gaïdar, interview dans EKO, 11, 1993.

10. C'est le cas notamment des philosophes ou sociologues anti-utilitaristes comme A. Caillé (cf. ses articles dans La Revue du MAUSS), P. Berger, Pyramids of Sacrifice, New York, Basic Books, 1974 ou R. Girard, La violence et le sacré, Paris, Hachette/Grasset, 1989.

11. Cette posture de délégation des intérêts au nom du bien commun, cette dépossession de soi en raison d'une auto-dépréciation et d'une méfiance à l'égard de ses propres élans, toutes ces diverses manifestations d'une aliénation de soi ont été 
constatées maintes fois parmi les travailleurs interrogés sur leur attitude à l'égard du politique.

12. La proportion était tombée à $20.8 \%$ fin 1997.

13. En termes réels, le salaire mensuel moyen a presque été divisé par deux de 1991 à 1997 (de 548 roubles à 296 roubles dans les prix de 1991).

14. La proportion des dépenses alimentaires dans le budget familial était de $28 \%$ en 1990. Elle est passée à 65-70\% en 1992, puis est retombée à 48-52\% en 1996-97. De plus, la qualité de l'alimentation se dégrade, celle-ci apportant moins de calories, de sels minéraux et de protéines.

15. Les évolutions de l'économie réelle ne se traduisent pas par une variation correspondante de la pauvreté mesurée officiellement, preuve d'une insuffisance ou d'un biais dans les méthodes.

En partie pour cette raison, des sociologues ont commencé, surtout à partir de 1994-95, à proposer des méthodes alternatives de mesure de la pauvreté[[La méthode officielle, utilisée par le Comité d'Etat aux Statistiques, procède par croisement entre le niveau de revenu déclaré dans les enquêtes et le seuil de pauvreté administrativement déterminé. Il s'agit de l'enquête Monitoring Longitudinale, méthode mise au point et adoptée en 1992 avec la collaboration de l'Université de Caroline du Nord, du Centre russe de Médecine Préventive, de l'Institut russe de Sociologie, avec le soutien financier de la Banque Mondiale et de l'Agence Américaine pour le Développement International. Voir T. Mroz et B. Popkins (ed.), Monitoring Economic Condition in russian Federation; The Russian Longitudinal Monitoring Survey 1992-1996, Chapen Hill, University of North Carolina, 1997. Une méthode similaire est appliquée par le Centre russe d'étude du niveau de vie, en partant d'un seuil de pauvreté plus élevé puisqu'il compte davantage de services et de produits non alimentaires. Les enquêtes sont effectuées avec le soutien d'un programe de l'ONU, voir United Nations Developement Programme (UNDP), Human Development Report for the Russian Federation, Moscou et New York, UNDP, 1997. Toutes ces approches, fortement inspirées par le modèle américain, sont fondées sur une définition absolue d'un seuil de pauvreté et s'attachent surtout à mesurer les revenus des ménages. Les alternatives à ces approches officielles mettent davantage l'accent sur l'appréciation subjective par les individus de leur niveau de vie. Certaines méthodes sont exclusivement subjectives et consistent à effectuer des enquêtes par questionnaires ou entretiens afin de cerner le « revenu minimum socialement acceptable dans les conditions actuelles de la société russe » tel qu'il « satisfasse les besoins vitaux de l'individu et préserve le respect de soi ». Cf. Bogatstvo, Bogatstvo i bednost' v Rossii, Moscou, 1996, p. 19. Des principes similaires inspirent les sondages effectués régulièrement par le Centre Russe d'Etude de l'Opinion Publique (VCIOM). De telles méthodes aboutissent à des appréciations du seuil de pauvreté supérieures de trois fois à celui du Comité Fédéral aux Statistiques. Voir son bulletin trimestriel, Monitoring obšcestvennogo mnenja. La sociologue T. Zaslavskaja se fonde ainsi souvent sur les sondages du VCIOM. De telles méthodes aboutissent à des appréciations du seuil de pauvreté supérieures de trois fois à celui du Comité Fédéral aux Statistiques. Contre le risque de subjectivisme, d'autres sociologues essaient encore de combiner différentes approches. Telle est, en particulier, la perspective affichée par une équipe de chercheurs de l'Institut d'étude des problèmes socio-économiques de la population. Cf. M.A. Moina et al., Bednost' : al'ternativnye podhody k opredeleniju i izmereniju, Moscou, Fondation Carnegie pour la Paix Internationale, 1998. Leurs enquêtes s'emploient à tenir compte à la fois des critères de revenu, des critères subjectifs (auto- 
positionnement dans la catégorie de « pauvres ») et des critères mi-subjectifs d'appréciation de son niveau de consommation (au travers du sentiment de subir des privations). Le croisement de ces différents critères les amènent à réviser à la baisse le seuil de pauvreté officiellement déterminé. L'intérêt de ce type d'étude ne réside d'ailleurs pas seulement dans cette révision, explicitement destinée à favoriser la mise en place d'une politique sociale plus sélective et réservée aux plus indigents. Il est aussi dans la mise en évidence de décalages entre la pauvreté des revenus, la pauvreté de la consommation et la pauvreté subjectivement vécue. Or, ces décalages ne se font pas toujours dans le même sens. Il arrive que des personnes se sentent pauvres tout en ayant un niveau de consommation moyen, mais l'inverse est tout aussi fréquent. Cette difficulté de cerner objectivement la pauvreté en Russie traduit l'absence de solidification du phénomène et rend peu pertinentes les tentatives d'identifier précisément des groupes sociaux qualifiables de « pauvres ».

16. Fin 1998, les estimations publiées par la FNPR (ex-fédération syndicale officielle) tournent autour de $80 \%$ (de la population au-dessous du seuil de pauvreté).

17. Ainsi T. Zaslavskaja met l'accent sur le taux de pauvreté de « l'intelligentisa de masse » (40\%), taux équivalent à celui de la masse des ouvriers de l'industrie, cf. Monitoring obšcestvennogo mnenja, n 6, 1995, pp. 11-13 ; L. Gordon insiste sur le fait « que l'augmentation de la pauvreté relative et absolue a affecté la partie la plus qualifiée des travailleurs, celle qui constitue le cœur de la classe moyenne des pays développés ", « Bednost' v sovremennom russkom obšcestve » [La pauvreté dans la société russe contemporaine], L.A. Beljaeva, Social'naja stratifikacija sovremennogo rossijskogo obšcestva v Rossii, Moscou, 1995, p. 61.

18. Ainsi, en 1995 , les $2 / 3$ des familles pauvres ont un chef de famille ayant un travail régulier.

19. Notre approche rejoint celle de chercheurs tels que B. Silverman et M. Yanowitch (cf. New Rich, New Poor, New Russia. Winners and Losers on the Russian Road to Capitalism, Armonk et Londres, M. E. Sharpe, 1996) ; ou de G. Standing (OIT) et T. Cetvernina (Centre d'étude du marché du travail auprès de l'Institut d'économie de l'Académie des Sciences de Russie), voir G. Standing, op.cit. et T. Tchetvernina, " Minimum Wages in Russia : Fantasy Chasing Fact », in G. Standing et D. VaughanWhitehead (ed.), Minimum Wages in Central and Eastern Europe : from protection to Destitution, Budapest et Londres, Central European Univertsity Press, 1995, pp. 49-67 ; ou encore celle de N.V. Cernina, "Social'nye problemy bezrabotnyh » [Les problèmes sociaux des chômeurs], Sociologiceskie issledovanija, nov. 1996, pp. 91-98.

20. Sur le secteur informel, cf. V. Gimpelson, « Rynok truda v castom sektore » [Le marché du travail dans le secteur privé], Predprinimatel'stvo v Rossii, 1, 1997, pp. 10-11; E. Klopov, «Vtoricnaja zanjatost' kak forma social'noj trudovoj mobil'nosti » [L'activité secondaire comme une forme de mobilité sociale et professionnelle], Sociologiceskie issledovanija, 4, 1997, pp. 29-45 ; K. Rousselet, « L'adaptation et la 'stratégie du contournement' », Cahiers Anatole Leroy-Beaulieu, juillet 1998, pp. 79-86. 21. Sur le secteur informel, cf. V. Gimpelson, « Rynok truda v castom sektore » [Le marché du travail dans le secteur privé], Predprinimatel'stvo v Rossii, 1, 1997, pp. 10-11; E. Klopov, «Vtoricnaja zanjatost' kak forma social'noj trudovoj mobil'nosti » [L'activité secondaire comme une forme de mobilité sociale et professionnelle], Sociologiceskie issledovanija, 4, 1997, pp. 29-45; K. Rousselet, « L'adaptation et la 'stratégie du contournement' », Cahiers Anatole Leroy-Beaulieu, juillet 1998, pp. 79-86. 22. Voir M.A. Moina et al, op. cit., p. 55 
23. Ibid., p. 56.

24. De nombreux écrits traduisent alors une telle sensibilité : « La structure de notre société n'est pas constituée de personnalités adultes avec leur individualisme (...) mais d'adolescents enclins à pratiquer le collectivisme, à se rassembler en grands groupes (...) où fonctionnent non la conscience individuelle et la responsabilité personnelle, mais des mécanismes d'identification idéologisée et des codes de comportements reflétant des règles éminemment arbitraires proclamées par les éléments les plus hautement placés dans la hiérarchie du pouvoir plutôt que des normes et principes universels », L. Sedov, « Russie : la voie étroite vers la démocratie », Etude, 337 (5), novembre 1992, pp. 447-458, p. 449 ; « Aujourd'hui, la société russe apparaît divisée en deux blocs (...). Le premier comprend les éléments parasites, incompétents, combinards (...) ; la psychologie lumpen y prédomine (...). L'autre bloc rassemble tous les éléments indépendants, actifs, entreprenants et capables », A. Yakovlev, « Conclusions à la Perestroïka ", Les Temps Modernes, janvier 1993, p. 85 ; « Je me risquerais à affirmer qu'un tel peuple n'a pas besoin de parlement, n'a pas besoin d'élection. Un tel peuple et ses élus (car on sait bien ce que peut élire un tel peuple) ne sont pas capables d'élaborer de nouvelles lois, de les rendre plus humaines, plus civilisées, plus raisonnables », F. Iskander, Stolica, n 5, 1994 (cité par A. Berelowitch, « Russie : l'avatar de l'idée démocratique ", Relations internationales et stratégiques, $n^{\circ} 14$, été 1994, pp. 50-54). 25. Ainsi V. Erofeev traite-t-il les « pauvres gens » : « (...) je n'ai pas de compassion pour toute cette triste masse qui tire la Russie vers le bas. L'attitude la plus simple à son égard est encore le sarcasme. (...) Mais, quelle que soit l'attitude que l'on adopte à l'égard de cette masse, le hasard l'a promue à la dignité d'électorat, elle dispose d'une voix et n'a rien de commun avec la démocratie. (...) Qu'en faire ? La duper ? La laver ? La rééduquer ? Attendre qu'elle meure ? (...) Il ne reste qu'à la parquer dans un camp de concentration. Mais, en fait, ils y sont déjà ", V. Erofeev, article paru dans Obšcaja Gazeta et traduit par Courrier International, sous le titre « En plein Moyen Age », 11-17 février 1999, p. 37.

26. Les médias font en effet une large place à cet intellectualisme et à ce démocratisme anti-populaire. Ils se montrent surtout féroces et accusateurs envers ceux qui, parmi la population en voie de paupérisation, n'acceptent pas passivement leur sort et tentent de résister par des actions collectives. Ainsi, à la veille des journées nationales d'action organisées par les syndicats du 27 mars 1997, les unes des journaux sont riches d'enseignements, en ce qu'elles tournent la plupart du temps en dérision les éventuels manifestants ou soulignent leur extrémisme et leur dangerosité. Certains journalistes agitent même le spectre de la guerre civile afin de dissuader les gens de participer aux actions de protestation. Seules quelques voix discordantes critiquent cet establishment " présomptueux et fabulateur » qui craint que « les gens simples, les travailleurs, piétinent la liberté pour un morceau de pain avec du saucisson », mais elles sont bien isolées. Si la crise d'août 1998 accélère la reconnaissance médiatique d'une pauvreté extensive, elle n'améliore pas le crédit de ceux qui manifestent leur refus de faire les frais d'une crise dont ils ne sont pas responsables. Lors de la journée nationale de protestation du 7 octobre 1998, les journalistes mettent ainsi l'accent sur les risques d'instrumentalisation et de récupération de la " juste colère du peuple » par des extrémistes. Ils tracent aussi des lignes de clivages entre les catégories sociales modestes mais décentes et les catégories pauvres et « hystériques».

27. Lors des opérations de blocage des voies ferrées par les mineurs en grève, au printemps 1998, le gouvernement s'est posé en donneur de leçons appelant les 
grévistes au sens de leurs responsabilités. Citons à titre d'exemple certains passages d'une interview accordée par N. Aksjenenko : «Je dirais que les mineurs se suicident en agissant ainsi. Leur vie dépend de la viabilité de notre branche. Les chemins de fer constituent le système sanguin qui irrigue tout le pays, et les mineurs aussi. Il est impossible de le malmener ainsi. (...) Quand il est apparu que l'action des mineurs se faisait au détriment de couches sociales encore plus démunies, qui n'ont même pas les moyens d'avoir accès aux transports les moins chers, d'acheter les produits et médicaments de première nécessité, alors la sympathie de l'opinion publique pour les mineurs s'est fortement érodée. Mais les gens commencent à comprendre que le problème doit se régler non sur les rails avec un Etat abstrait, pas même avec le président du pays, mais avec les employeurs et les actionnaires. A cause de l'incapacité des mineurs à régler eux-mêmes leurs affaires, tous souffrent. (...) Ils perdent des marchés, et nous aussi, seulement parce qu'ils veulent satisfaire leurs ambitions, sacrifiant les intérêts des autres gens. (...) Ils rusent, ils rusent énormément. Dans leurs protestations, il y a plus de parasitisme, caractéristique de l'époque soviétique, que de sens des responsabilités devant leurs familles qui n'ont plus rien aujourd'hui pour se nourrir, en grande partie à cause de leur courte vue (...) Rappelons-nous que la majorité des mineurs ont accueilli la privatisation avec joie. Le problème est que l'homme moderne a obtenu la possibilité de déterminer lui-même son destin et de ne pas dépendre de l'Etat, mais il ne s'est pas révélé prêt à faire cela », N. Aksjenenko : « ...Ja by šahterov nazval samoubijcami » [Je qualifierais les mineurs de suicidaires], Moskovskie Novosti, 24-31 mai 1998, pp. 2-3.

28. Il n'est pas insignifiant que cette façon de penser et de présenter la pauvreté se soit accompagnée d'une publicisation inédite de propos antisémites. Si l'antisémitisme n'a rien en soi de nouveau, il est aujourd'hui exploité par les médias et la classe politique libérale dans le but de discréditer les « revanchards » et d'agiter la menace d'une dérive extrémiste de la population « lumpenarisée ».

29. Ces propos ont été recueillis dans toutes les entreprises et la plupart des ateliers, mais beaucoup plus dans celles et ceux qui offrent un environnement de travail relativement favorable à une construction subjective de son individualité.

30. R. Castel, Les métamorphoses de la question sociale. Une chronique du salariat, Paris, Fayard, 1995, p. 465 : «Individualisme négatif parce qu'il se décline en termes de manque - manque de considération, manque de sécurité, manque de biens assurés et de liens stables ".

31. « La part des ménages se situant en deçà du seuil de pauvreté (...) place 60 à $70 \%$ de la population dans une situation de grande détresse. Il n'est donc pas étonnant de reformuler la définition du seuil de pauvreté pour le rendre plus conforme aux nouvelles conditions de vie des Russes. Il était en effet urgent de distinguer, dans la masse de la population appauvrie, les plus démunis réclamant une attention particulière (...) », N. Rimachevskaïa, « Paupérisation et exclusion en Russie », Le Courrier des Pays de l'Est, juillet 1998, pp. 58-99, p. 58.

32. N.V. Cernina, adepte de la notion d'exclusion, indique l'importance qu'a eu le forum sur les politiques sociales contre l'exclusion, tenu au siège de l'ONU en mai 1996, cf. Cernina, Social'nye problemy bezrabotnyh, op.cit., p. 92 ; dans un rapport pour la Banque Mondiale, J. Klugman et J. Braithwaith notent que « le débat européen sur l'exclusion sociale de groupes marginalisés peut être transposé à la Russie ", cf. "Poverty in Russia during the Transition : An Overview », The Worl Bank Observer, 1 (13), feb. 1998, pp. 37-58, p. 55. 
33. Quelle inflexion de sens introduit la notion d'exclusion par rapport à celle de pauvreté ? En premier lieu, elle met davantage l'accent sur un état d'extériorité au regard de la société, des rapports sociaux en général. A contrario, s'estompe quelque peu la dimension d'inégalité sociale contenue dans la notion de pauvreté. Les rapports sociaux de domination se dissolvent dans le concept d'exclusion qui divise la société horizontalement (les « in » et les « out») et non verticalement. Cf. Alain Touraine, "L'affaire n'est plus aujourd'hui d'être 'up or down' mais 'in or out' », in Jacques Donzelot et Joël Roman (dir.), Face à l'exclusion, le modèle français, Paris, éd. Esprit, 1991, p. 8. Déjà critiqué en France par de nombreux sociologues (voir en particulier R. Castel, op. cit. ; Y. Clot, Le travail sans l'homme ?, Paris, La Découverte, 1995 ; P. Rolle, Où va le salariat?, Lausanne, Ed. Page deux, 1997), le concept doit a fortiori être interrogé dans le cas de la Russie. La majorité des dits « exclus » se trouvent en effet être des travailleurs, insérés comme tels dans des rapports sociaux de domination, qu'ils soient d'employeurs à employés ou de chefs de réseau informel à exécutants, de travailleurs à détenteurs du capital matériel, technique et surtout financier. Or, parler d'exclusion, c'est aussi faire dévier le regard du cœur du problème, à savoir le travail, vers la périphérie, c'est-à-dire la marginalisation, la sortie hors société ou hors travail. En deuxième lieu, la notion d'exclusion introduit une différenciation au sein de l'ensemble des " pauvres ", potentiellement source de divisions, voire de conflits entre ceux qui seraient jugés plus ou moins démunis, plus ou moins intégrés, plus ou moins qualifiés pour profiter des aides sociales. Or, la situation est bien moins figée. Il est impossible de tracer une frontière entre pauvres et exclus, les populations des deux catégories interchangeant constamment leurs positions et aucune condition sociale n'étant assurée, même pas la pire. Enfin, une analyse en terme d'exclusion multiplie les risques de stigmatisation des victimes car, si le système socio-institutionnel produit en partie l'exclusion, celle-ci résulte aussi pour une part de l'inaptitude des exclus à s'intégrer, ce qui se traduit en Russie par inadaptation aux changements, à l'économie de marché, incapacité à se défaire des anciennes façons d'être, évoquées en des termes exclusivement dépréciatifs, quand les exclus ne sont pas tout simplement criminalisés. 34. Observant les résultats des réformes quelques années après leur introduction, en particulier à partir de 1994, de nombreux sociologues ont déchanté en constatant leur impact destructeur sur les travailleurs, cf. B. Silverman et M. Yanowitch, op. cit. 35. Le ministre du travail, G. Melikian, affirme ainsi en mars 1996 : « La Russie doit passer des slogans idéologiques sur la lutte des classes au partenariat social (...). L'étape de prostration et de choc est en train de passer, commence maintenant la période de l'optimisation de la production, ce qui nécessite une diminution du nombre de travailleurs en trop ». Cf. « Kto zašcitit rabocego ? » [Qui défendra l'ouvrier ?], Trud, 20.03.96.

36. Voir, par exemple, P. Kapeljušnikov, « Otkuda 'lišnie' ljudi » [Qui sont les « gens en trop »], Ekonomika i izn', n 19, mai 1998 (les guillemets n'empêchent pas ce chercheur de l'IMEMO de présenter les caractéristiques de ces « gens en trop »).

37. Ainsi une enquête dont les résultats figurent dans le bulletin du VCIOM, I. Perova, « Izbytocnaja zanjatost' i ugroza sokrašcenij na predprijatijah razlicnyh otraslej promyšlennosti » [Le surplus de main-d'œuvre et la menace de licenciements dans les entreprises de différentes branches industrielles], Monitoring obšcestvennogo mnenija, $\mathrm{n}^{\circ}$ 5, septembre-octobre 1997, pp. 24-26. 
38. Par exemple A. Moskovskaja, « Izbytocnaja zanjatost' na promyšlennyh predprijatijah Rossii : pro i contra » [Le surplus de main-d'oeuvre dans les entreprises industrielles de Russie : le pour et le contre], Voprosy Ekonomiki, n 1, 1998, pp. 59-72.

INDEX

Index géographique : Russie

Mots-clés : pauvreté, exclusion 